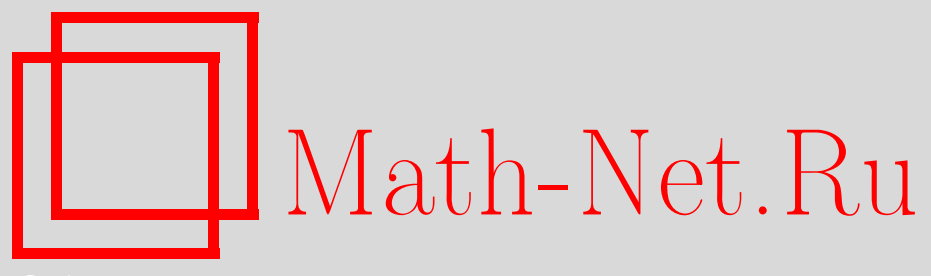

А. Р. Алимов, Монотонная линейная связность и солнечность связных по Менгеру множеств в банаховых пространствах, Изв. РАН. Сер. матем., 2014, том 78, выпуск 4, 3-18

DOI: https://doi.org/10.4213/im8128

Использование Общероссийского математического портала Math-Net.Ru подразумевает, что вы прочитали и согласны с пользовательским соглашением http://www.mathnet.ru/rus/agreement

Параметры загрузки:

IP: 54.237 .206 .68

26 апреля 2023 г., $14: 36: 48$

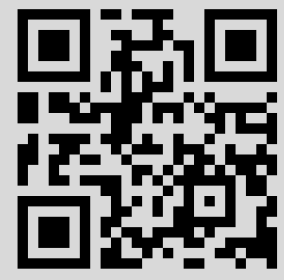




\title{
А. Р. Алимов \\ Монотонная линейная связность и солнечность связных по Менгеру множеств в банаховых пространствах
}

\begin{abstract}
Установлено, что в широком классе банаховых пространств (в частности, в сепарабельных) ограниченно компактное m-связное (связное по Менгеру) множество монотонно линейно связно и является солнцем. Показано, что пересечение ограниченно компактного монотонно линейно связного (m-связного) множества с замкнутым шаром клеточноподобно (имеет шейп точки), в частности ациклично (в конечномерном случае - стягиваемо), и является солнцем и что ограниченно слабо компактное m-связное множество монотонно линейно связно. Попутно теорема Рейнуотера-Симонса о слабой сходимости последовательностей распространяется на случай сходимости относительно ассоциированной (по Брауну) нормы.
\end{abstract}

Библиография: 38 наименований.

Ключевые слова: солнце, ацикличное множество, клеточноподобное множество, монотонно линейно связное множество, связность по Менгеру, $d$-выпуклость, выпуклость по Менгеру, теорема Рейнуотера-Симонса.

DOI: $10.4213 / \operatorname{im} 8128$

\section{§ 1. Введение и основные определения}

Для ограниченного подмножества $M \neq \varnothing$ действительного линейного нормированного пространства $X$ через $\mathrm{m}(M)$ обозначается пересечение всех замкнутых шаров, содержащих $M$ (следуя [1], $\mathrm{m}(M)$ называется оболочкой Бана$x a-$ Мазура (шаровой оболочкой) множества $M$ ). Множество $M \subset X$ называем m-связным (связным по Менгеру) [1], если $\mathrm{m}(\{x, y\}) \cap M \neq\{x, y\}$ для любых $x, y \in M$. Для краткости далее обозначаем $\mathrm{m}(\{x, y\})=\mathrm{m}(x, y)$. Несмотря на название, в бесконечномерном случае замкнутое m-связное множество не обязано быть связным.

Пусть $k(\tau), 0 \leqslant \tau \leqslant 1,-$ непрерывная кривая в линейном нормированном пространстве $X$. Следуя [2], говорим, что кривая $k(\cdot)$ монотонна, если $f(k(\tau))$ является монотонной функцией по $\tau$ для любого $f \in \operatorname{ext} S^{*}$ (здесь и далее ext $S^{*}$ - множество крайних точек сферы $S^{*}$ сопряженного пространства).

Замкнутое множество $M$ называется монотонно линейно связным [3], если любые две точки из $M$ можно соединить непрерывной монотонной кривой (дугой) $k(\cdot) \subset M$.

Естественность m-связных множеств и монотонно линейно связных множеств при изучении связности солнц была показана в работах [1], [3]-[5]: для

Работа выполнена при финансовой поддержке РФФИ (грант № 13-01-00022).

(C) А.Р. Алимов, 2014 
монотонно линейно связного солнца удается ответить на давно поставленный вопрос о связности (и даже ацикличности) пересечения солнца с шаром, что существенно дополняет известную теорему Л. П. Власова о солнечности ацикличных множеств.

Для $x \in X$ и $\varnothing \neq M \subset X$ посредством $P_{M} x$ мы обозначаем метрическую проекцию на множество $M$ (множество ближайших точек из $M$ к $x$ ).

Подмножество $M \neq \varnothing$ линейного нормированного пространства $X$ называется солнием [6], если для каждой точки $x \in X \backslash M$ существует точка $y \in P_{M} x$ (точка светимости) такая, что

$$
y \in P_{M}[(1-\lambda) y+\lambda x] \quad \forall \lambda \geqslant 0 .
$$

Понятие солнца было введено Н.В. Ефимовым и С.Б. Стечкиным в 1958 г. при изучении чебышевских множеств, при этом под солнцем они понимали то, что сейчас называют строгим протосолнцем (такие множества $M$ определяются тем, что (1.1) выполнено для любых $x \notin M$ и $\left.y \in P_{M} x\right)$.

Отметим [7], что чебышевское монотонно линейно связное множество всегда является солнцем; более того, если $M$ - монотонно линейно связное множество и $P_{M} x=\{y\}, x \notin M$, то $y$ - точка светимости. Из определения вытекает, что монотонная линейная связность сохраняется при пересечении монотонно линейно связного множества с экстремальной гиперплоскостью и, более того, с произвольным брусом (т. е. множеством, образованным пересечениями гиперполос, порожденных экстремальными точками сопряженной сферы [8]). Поскольку замкнутый шар является брусом, монотонно линейно связное множество необходимо $B$-монотонно линейно связно, т. е. его пересечение с любым замкнутым (а следовательно, и открытым) шаром монотонно линейно связно. Отсюда вытекает [5], что монотонно линейно связное множество т-связно. Обратное утверждение неверно - соответствующий пример предложен в [9]: $M=M_{1} \cup M_{2}$, где $M_{i}=\{x \in C[0,1] \mid x(0)=i, i=1,2\}$. Однако для замкнутых множеств в $c_{0}$ (см. [4]) и для ограниченно компактных множеств в произвольном сепарабельном пространстве $X$ эти свойства эквивалентны (см. далее теорему 4.1).

В работе изучаются топологические и аппроксимативные свойства m-связных (связных по Менгеру) множеств и монотонно линейно связных множеств, исследуется их солнечность. В теореме 4.1 утверждается, что в широком классе банаховых пространств (в частности, в сепарабельных пространствах) ограниченно компактное m-связное множество монотонно линейно связно и является солнцем. При этом пересечение ограниченно компактного монотонно линейно связного множества с замкнутым шаром клеточноподобно (имеет шейп точки), в частности ациклично, и является солнцем. Этот результат частично дополняет известную теорему Власова [6], согласно которой ограниченно компактное $P$-ацикличное подмножество банахова пространства является солнцем. Случай слабо компактных множеств рассматривается в теореме 4.2. При доказательстве теоремы 4.1 мы попутно обобщаем известную теорему Рейнуотера-Симонса о слабой сходимости на случай сходимости относительно ассоциированной нормы (предложение 3.1 ). 


\section{§ 2. Ацикличные и клеточноподобные множества}

Теория гомологий (когомологий) связывает с каждым топологическим пространством $X$ последовательности абелевых групп $H_{k}(X), k=0,1,2, \ldots$ (группы гомологий), и $H^{k}(X), k=0,1,2, \ldots$ (группы когомологий), которые являются гомотопическими инвариантами пространства: если два пространства гомотопически эквивалентны, то и соответствующие группы гомологий изоморфны. Конструировать группы гомологий (когомологий) можно различными способами, из которых мы можем выделить следующие: конструкция, основанная на нервах покрытий, предложенная П. С. Александровым и обобщенная Э. Чехом; конструкция Л. Вьеториса, основанная на понятии истинных циклов и применимая к метрическим пространствам; конструкция, основанная на понятии сингулярных цепей (непрерывных образов симплициальных цепей).

Пусть $A$ - произвольная нетривиальная абелева группа. Пространство (далее все пространства предполагаются метризуемыми) называется ащикличным, если его группа чеховских когомологий с коэффициентами из $A$ тривиальна (не имеет циклов, за исключением границы). Таким образом, определение ацикличности зависит от выбранной группы коэффициентов. Здесь отметим, что гомологии Александрова-Чеха не образуют теории гомологий, не удовлетворяя аксиоме точности, а когомологии Чеха образуют теорию гомологий топологических пространств. С подробным изложением теории гомологий (когомологий) компактов, топологических и равномерных пространств можно ознакомиться в обзоре С. А. Мелихова [10].

В случае, если гомология (когомология) имеет компактный носитель (т. е. удовлетворяет аксиоме компактных носителей) и коэффициенты группы гомологий (когомологий) лежат в поле, то понятия гомологической и когомологической ацикличности совпадают [11]. Однако в случае произвольной абелевой группы коэффициентов понятия гомологической и когомологической ацикличности могут быть различны. K примеру, 2-адический соленоид (предел обратной последовательности $S^{1} \stackrel{f}{\leftarrow} S^{1} \stackrel{f}{\leftarrow} \cdots$, где $f=z^{2}$ ) ацикличен в гомологиях Чеха с коэффициентами в поле $\mathbb{Z}_{2}$, а в когомологиях Чеха ацикличности нет (см., например, [12]).

Далее, если не оговорено противное, ацикличность будет пониматься относительно чеховских когомологий с коэффициентами в произвольной абелевой группе.

Непустое компактное пространство называется $R_{\delta}$-множеством (см., например, $[13,(2.11)])$, если оно гомеоморфно пересечению счетной убывающей последовательности абсолютных ретрактов (или стягиваемых компактов [13, теорема 2.13]). $R_{\delta}$-множества естественно возникают как пространства решений задачи Коши, а также автономных и неавтономных дифференциальных включений [14]-[16]. Результаты такого типа восходят к работам Ароншайна.

Компакт $Y$ называется клеточноподобным (имеющим шейп точки), если существует ANR (абсолютный окрестностный ретракт) $Z$ и вложение $i: Y \rightarrow Z$ такое, что образ $i(Y)$ стягиваем в любой своей окрестности $U \subset Z$ (см. [13, $(82.4)])$; само клеточноподобное множество при этом не обязано быть стягиваемым. Из известной характеризации Химана $R_{\delta}$-множеств непосредственно следует, что $R_{\delta}$-множество всегда клеточноподобно $[17, \S 4.2],[18$, с. 50$]$. Поскольку всякое отображение компакта точечного шейпа в ANR гомотопически тривиально, компакт шейпа точки (клеточноподобный) стягивается во всякой своей 
окрестности в любом объемлющем ANR. Как следствие, $к л а с с ы ~ R{ }_{\delta}$-множеств и клеточноподобных (шейпа точки) компактов совпадают.

Отметим, что клеточноподобность влечет ацикличность (относительно любой непрерывной теории (ко)гомологий) [17, с. 854], при этом имеются примеры ацикличных, но не клеточноподобных множеств, а также клеточноподобных, но не линейно связных множеств (синусоида топологов).

Следуя [6], если Q обозначает некоторое свойство (например, “связность"), мы будем говорить, что множество $M$ обладает одним из следующих свойств:

$P$-Q, если при всех $x \in X$ множество $P_{M}(x)$ непусто и обладает свойством $\mathrm{Q}$;

$B$-Q, если $M \cap B(x, r)$ обладает свойством Q при всех $x \in X, r>0$;

$\stackrel{\circ}{B}$-Q, если $M \cap \stackrel{\circ}{B}(x, r)$ обладает свойством Q при всех $x \in X, r>0$.

Таким образом, замкнутое подмножество конечномерного пространства $P$-непусто или является множеством существования (проксиминально). Соответственно, множество $M P$-ациклично, если $P_{M} x$ непусто и ациклично для любого $x$; множество $M \quad B$-ациклично, если пересечение $M$ с любым замкнутым шаром ациклично.

ЗАмечАниЕ 2.1. $B$-связные множества также называют $V$-связными (здесь буква $V$ происходит из работ Л.П. Власова, который обозначал шары как $V(x, r))$. Наш термин $B$-связность согласуется с более привычным обозначением шаров как $B(x, r)$, а также с понятием "ограниченная связность" (bounded connectedness), введенным Д. Вулбертом в 1960-х годах.

А.Л. Браун [19, следствие 1.6.2] доказал, что если ограниченно компактное подмножество $M$ банахова пространства $P$-ациклично (относительно чеховских когомологий с коэффициентами в произвольной абелевой группе), то $M B$-ациклично ${ }^{1}$. Таким образом, ацикличность произвольного компактного $\mathrm{m}$-связного множества влечет $P$ - и $B$-ацикличность произвольного ограниченно компактного $\mathrm{m}$-связного множества $M$. Это следует из того, что пересечение такого $M$ с произвольным шаром $B(x, r)$ компактно и т-связно.

\section{§ 3. Оболочка Банаха-Мазура. Монотонная линейная связность. Классы пространств (MeI) и (Ex- $\left.w^{*} \mathrm{~s}\right)$. Ассоциированная норма. Теорема Рейнуотера-Симонса}

В настоящем параграфе изучается связь между введенной выше оболочкой Банаха-Мазура $\mathrm{m}(\cdot, \cdot \cdot)$ и (функциональным) интервалом $\llbracket \cdot, \cdot \rrbracket($ определенным далее в (3.2)). Вводится класс (MeI) линейных нормированных пространств (включающий все сепарабельные), в котором $\mathrm{m}(\cdot, \cdot)=\llbracket \cdot, \cdot \rrbracket$. Важным свойством оболочки Банаха-Мазура $\mathrm{m}(\cdot, \cdot)$ является то, что $z \in \mathrm{m}(x, y)$, если и только если $z$ лежит метрически между $x$ и $y$ относительно так называемой ассоциированной (по Брауну) нормы $|\cdot|$. Такая норма вводится на пространствах класса $\left(\mathrm{Ex}-w^{*} \mathrm{~s}\right)$, который также включает в себя все сепарабельные банаховы пространства. Данное обстоятельство позволяет привлечь аппарат метрической выпуклости (лемма 5.А). Попутно мы распространяем известную теорему Рейнуотера-Симонса о слабой сходимости последовательностей на случай сходимости относительно ассоциированной нормы (предложение 3.1).

\footnotetext{
${ }^{1}$ На самом деле Браун показал в [19] несколько больше: если $M-P$-ацикличное аппроксимативно компактное подмножество банахова пространства и пересечение $M$ с некоторым шаром $B$ компактно, то $M \cap B$ ациклично.
} 
Для начала отметим, что в пространстве $X=C(Q)$ структура $\mathrm{m}(x, y)$, $x, y \in X$, вполне ясна:

$$
\mathrm{m}(x, y)=\llbracket x, y \rrbracket
$$

где

$$
\llbracket x, y \rrbracket:=\{z \in C(Q) \mid z(t) \in[x(t), y(t)] \quad \forall t \in Q\} .
$$

Аналогичное представление также верно и в пространстве $C_{0}(Q)$ (где $Q$ - локально компактное хаусдорфово пространство), это следует из характеризации [20] крайних точек ("значение в точке") единичной сферы сопряженного пространства к $C_{0}(Q)$.

В общем случае по аналогии с (3.1) интервал $x, y \rrbracket$ определяется выражением

$$
\llbracket x, y \rrbracket:=\left\{z \in X \mid \min \{\varphi(x), \varphi(y)\} \leqslant \varphi(z) \leqslant \max \{\varphi(x), \varphi(y)\} \quad \forall \varphi \in \operatorname{ext} S^{*}\right\} .
$$

Следуя [1], а также [9], введем в рассмотрение класс пространств $X$, который мы обозначаем

$$
(\mathrm{MeI}): \mathrm{m}(x, y)=\llbracket x, y \rrbracket \quad \forall x, y \in X .
$$

Сокращение (MeI) происходит от английского "The hull $\mathrm{m}(x, y)$ equals the interval $\llbracket x, y \rrbracket$ for all $x, y$ ”. Автору не известны банаховы пространства, не лежащие в классе (MeI).

Заметим, что включение $\mathrm{m}(x, y) \supset \llbracket x, y \rrbracket$ имеет место в любом линейном нормированном пространстве $X$ (см., например, [5]). Также известно [5], [9], что равенство $\mathrm{m}(x, y)=\llbracket x, y \rrbracket$ имеет место для достаточно широкого класса пространств и, в частности, для пространств, на единичной сфере которых гладкие точки всюду плотны (такой класс содержит слабо асплундовы, а следовательно, и сепарабельные пространства).

Несложно показать, что класс (MeI) содержит пространства $C(Q)$, где $Q-$ хаусдорфов компакт, и, в частности, пространство $\ell^{\infty}$ (как пространство непрерывных функций на стоун-чеховской компактификации натурального ряда). Также отметим, что если пространство $X$ таково, что ext $S^{*}$ лежит в замыкании множества $w^{*}$-полуострых точек шара $B^{*}$ (условие Морено), то $\llbracket x, y \rrbracket=\mathrm{m}(x, y)$ для всех $x, y \in X$; такому условию, в частности, удовлетворяют конечномерные пространства и пространства со свойством пересечения Мазура. Напомним, что точка $f \in S^{*}$ называется $w^{*}$-nолуострой точкой сопряженного шара $B^{*}$ (см., например, [21]), если для любого $\varepsilon>0$ найдется $w^{*}$-срез Sl шара $B^{*}$ такой, что $\operatorname{diam}(\{f\} \cup \mathrm{Sl})<\varepsilon$. Здесь $\operatorname{Sl}\left(B^{*}, x, \delta\right):=\left\{g \in S^{*} \mid g(x)>1-\delta\right\}$, $0<\delta<1, x \in X$.

Далее также понадобится класс пространств

$$
\left(\mathrm{Ex}-w^{*} \mathrm{~s}\right): \operatorname{ext} S^{*} w^{*} \text {-сепарабельно, }
$$

введенный Франчетти и Роверси [9]. При этом в определении класса (Ex- $\left.w^{*} \mathrm{~s}\right)$ мы всегда предполагаем, что

$$
F=\left(f_{i}\right)_{i \in I} \subset \operatorname{ext} S^{*} \quad w^{*} \text {-плотно в } \operatorname{ext} S^{*}, \quad \operatorname{card} I \leqslant \aleph_{0}, \quad F=-F .
$$

Сокращение $\left(\mathrm{Ex}-w^{*} \mathrm{~s}\right)$ происходит от немецкого "Die Extrempunktmenge der konjugierten Einheitskugel ist $w^{*}$-separabel". 
Из теоремы Крейна-Мильмана сразу следует, что любое пространство из класса $\left(\mathrm{Ex}-w^{*} \mathrm{~s}\right)$ имеет $w^{*}$-сепарабельный единичный шар $B^{*}$. Последнее условие эквивалентно [22] тому, что $X$ изометрично изоморфно подпространству из $\ell^{\infty}$. Также отметим, что имеются примеры пространств типа $X=C(K)$, где $K$ - несепарабельный хаусдорфов компакт, или $X=\ell_{1} \oplus \ell_{2}(\Gamma),|\Gamma|=\mathfrak{c}$, для которых $X^{*} w^{*}$-сепарабельно, а единичный шар $B^{*}$ не $w^{*}$-сепарабелен.

Далее, если $X$ - сепарабельное линейное нормированное пространство, то $w^{*}$-топология единичного шара $B^{*}$ сопряженного пространства $X^{*}$ метризуема. Соответственно, шар $B^{*} w^{*}$-сепарабелен [25, следствие 3.104]. Поэтому любое сепарабельное пространство лежит в классе (Ex-w*s). Также отметим, что класс $\left(\mathrm{Ex}-w^{*} \mathrm{~s}\right)$ содержит несепарабельное пространство $\ell^{\infty}$ (как пространство непрерывных функций на стоун-чеховской компактификации натурального ряда $\beta \mathbb{N}$, данный компакт сепарабелен, но не метризуем). При этом $C(Q)$ на несепарабельном $Q$ и $c_{0}(\Gamma)$ на несчетном $\Gamma$ не лежат в $\left(\mathrm{Ex}-w^{*} \mathrm{~s}\right)$.

Было бы интересно получить характеризацию пространств класса (Ex- $\left.w^{*} \mathrm{~s}\right)$.

Кратко суммируя сказанное выше относительно классов пространств (MeI) и $\left(\mathrm{Ex}-w^{*} \mathrm{~s}\right)$, отметим, что класс $(\mathrm{MeI}) \cap\left(\mathrm{Ex}-w^{*} \mathrm{~s}\right)$ содержит все сепарабельные линейные нормированные пространства (в частности, все пространства $C(Q)$ на метризуемом компакте $Q$ ) и несепарабельное пространство $\ell^{\infty}$.

Пусть $X \in\left(\mathrm{Ex}-w^{*} \mathrm{~s}\right), F=\left(f_{i}\right)_{i \in I}-$ семейство функционалов из $\left(\mathrm{Ex}-w^{*} \mathrm{~s}\right)$, $\left(\alpha_{i}\right) \subset \mathbb{R}, \alpha_{i}>0, i \in I$, и пусть $\sum \alpha_{i}<\infty$. Для $x \in X$ положим

$$
|x|=\sum_{i \in I} \alpha_{i}\left|f_{i}(x)\right| .
$$

Тогда $|\cdot|$ - норма на $X$, которую, следуя [1], мы называем ассоииированной. Ясно, что $|x| \leqslant\|x\| \sum \alpha_{i}$.

Важность ассоциированной нормы показывает следующий результат, который является естественным и простым обобщением следствия 3.2 из [1], доказанного Брауном в случае $\operatorname{dim} X<\infty$.

Лемма 3.1. Пусть $X$ - банахово пространство из класса $(\mathrm{MeI}) \cap\left(\mathrm{Ex}-w^{*} \mathrm{~s}\right)$ (в частности, $X$ - сепарабельное банахово пространство), и пусть $x, y \in X$. Следующие условия эквивалентны:

a) $z \in \mathrm{m}(x, y)$

b) $\left|f_{i}(x)-f_{i}(y)\right|=\left|f_{i}(x)-f_{i}(z)\right|+\left|f_{i}(z)-f_{i}(y)\right|$ для всех $i \in I$, где $F=\left(f_{i}\right)_{i \in I}-$ семейство из определения класса $\left(\mathrm{Ex}-w^{*} \mathrm{~s}\right)$;

c) $|x-y|=|x-z|+|z-y|$.

ДокАЗАтельство. Пусть $z \in \mathrm{m}(x, y)$. Поскольку $X \in(\mathrm{MeI})$, по определению имеем $f(z) \in[f(x), f(y)]$ для любого $f \in \operatorname{ext} S^{*}$. В частности, выполнено условие b) и в силу (3.3) условие с). Обратно, пусть $z$ находится между $x$ и $y$ относительно нормы $|\cdot|$. Ясно, что $\left|f_{i}(x-y)\right| \leqslant\left|f_{i}(x-z)\right|+\left|f_{i}(z-y)\right|$. Отсюда и из (3.3) имеем

$$
\left|f_{i}(x)-f_{i}(y)\right|=\left|f_{i}(x)-f_{i}(z)\right|+\left|f_{i}(z)-f_{i}(y)\right|
$$

для всех $i$. Как следствие,

$$
|f(x)-f(y)|=|f(x)-f(z)|+|f(z)-f(y)|
$$

для всех $f \in \operatorname{ext} S^{*}$, поскольку $\left(f_{i}\right) w^{*}$-плотно в ext $S^{*}$. Окончательно имеем $z \in \mathrm{m}(x, y)$ в силу того, что $X \in(\mathrm{MeI})$. 
Следующий результат можно рассматривать как распространение известной теоремы Рейнуотера-Симонса (см., например, [25, §3.11.8.5]) на случай сходимости относительно ассоциированной нормы $|\cdot|$ на пространствах класса $\left(\mathrm{Ex}-w^{*} \mathrm{~s}\right)$ (в частности, на сепарабельных пространствах). Теорема РейнуотераСимонса утверждает, что ограниченная последовательность $\left(x_{n}\right)$ в банаховом пространстве $X$ слабо сходится к $x \in X$, если и только если последовательность $\left(f\left(x_{n}\right)\right)$ сходится к $f(x)$ для каждого функционала $f$ из произвольной фиксированной границы Джеймса пространства $X$ (например, для всех $f \in \operatorname{ext} S^{*}$ ). Таким образом, хотя в общем случае слабая сходимость не метризуема, имеется норма на $X \in\left(\mathrm{Ex}-w^{*} \mathrm{~s}\right)$, относительно которой сходимость последовательностей равносильна слабой сходимости.

Напомним (см., например, $[25, \S 3.11 .8]$ ), что подмножество $A$ единичной сферы $S^{*}$ сопряженного пространства $X^{*}$ называется границей (Джеймса) для пространства $X$, если для каждого $x \in X$ найдется $f \in A$ такой, что $f(x)=\|x\|$. Из теоремы Крейна-Мильмана легко вытекает, что множество ext $S^{*}$ крайних точек сопряженного шара является границей для $X$.

ПредлоЖЕнИЕ 3.1. Пусть $X \in\left(\mathrm{Ex}-w^{*} \mathrm{~s}\right)$ - банахово пространство, $F:=$ $\left(f_{i}\right)_{i \in I} \subset \operatorname{ext} S^{*}$ - набор функиионалов из определения класса (Ex-w*s). Пусть $\left(x_{n}\right)$ - ограниченная последовательность в X. Рассмотрим следующие условия:

a) $x_{n} \stackrel{|\cdot|}{\longrightarrow} x$

b) $f_{i}\left(x_{n}\right) \rightarrow f_{i}(x)$ для любого $i \in I$;

c) $x_{n} \stackrel{w}{\longrightarrow} x$.

Тогда условия а) и b) эквивалентны, любое из них обеспечивается выполнением условия с). Если $X^{*}$ сепарабельно, то условия а)-с) эквивалентны.

ЗАмечАниЕ 3.1. В импликации b) $\Rightarrow$ c) сепарабельность $X^{*}$ существенна. Действительно, пусть $X=\ell^{1}$. Рассмотрим финитные последовательности из $\ell^{\infty}$, состоящие из нулей и плюс-минус единиц. Таких последовательностей счетное число, и они $w^{*}$-плотны во множестве ext $S^{*}$. Однако из сходимости элементов из $\ell^{1}$ на этих финитных последовательностях не следует их слабая сходимость. Данный факт отмечен П. А. Бородиным при обсуждении результатов работы.

ЗАмечАнИЕ 3.2. Из предложения 3.1 вытекает, что пространство $(X,|\cdot|)$ всегда является пространством Шура относительно ассоциированной нормы $|\cdot|$ (пространство называется пространством Шура, если в нем сходимость последовательности по норме равносильна ее слабой сходимости; классическим примером такого пространства является пространство $\left.\ell^{1}\right)$. Действительно, из определения ассоциированной нормы следует, что $|x|>\alpha_{i}\left|f_{i}(x)\right|$ для любого $i$, откуда вытекает, что любой элемент $f_{i} \in F$ лежит в $X_{|\cdot|}^{*}$. Теперь из условия b) предложения 3.1 следует, что если $\left(x_{n}\right)$ сходится, то $\left(x_{n}\right)|\cdot|$-сходится. Таким образом, для последовательности $\left(x_{n}\right)$

$$
x_{n} \stackrel{w_{|\cdot|}}{\longrightarrow} x \Longleftrightarrow x_{n} \stackrel{|\cdot|}{\longrightarrow} x .
$$

В результате имеем, что в пространстве $(X,|\cdot|) w_{|\cdot|}$-компактность совпадает с сильной $|\cdot|$-компактностью, и, как следствие, если $(X,|\cdot|)$ рефлексивно, то оно конечномерно. 
Данное замечание возникло в результате переписки с О. Нигардом, которому автор выражает искреннюю благодарность.

ДоКАЗАТЕЛЬСТво ПРЕДЛОЖЕНИЯ 3.1. Импликация а) $\Rightarrow$ b) вполне понятна: если $x_{n} \stackrel{|\cdot|}{\longrightarrow} x$ (по норме $\left.|\cdot|\right)$, то сумма $\sum \alpha_{i}\left|f_{i}\left(x_{n}\right)-f_{i}(x)\right|$ мала при всех достаточно больших $n$; как следствие, для каждого фиксированного $j$ разность $\left|f_{j}\left(x_{n}\right)-f_{j}(x)\right|$ также мала при таких $n$.

Установим b) $\Rightarrow \mathrm{a})$. Для каждого $n$ разобьем сумму $\sum_{i \in I} \alpha_{i}\left|f_{i}\left(x_{n}\right)-f_{i}(x)\right|$ на две: при $i \leqslant N$ и при $i>N$ ( $N$ выберем позже). Поскольку по условию последовательность $\left(x_{n}\right)$ равномерно ограничена, то во второй сумме выполнено $\left|f_{i}\left(x_{n}\right)-f_{i}(x)\right| \leqslant C$ (где $C$ не зависит от $\left.i, n\right)$. Как следствие, вторая сумма ограничена сверху суммой $\sum_{i>N} C \alpha_{i}<\infty$. По $\varepsilon>0$ выбираем $N$, при котором вторая сумма меньше $\varepsilon$. Первая сумма конечная, и в ней выбираем большие $n$.

Импликация c) $\Rightarrow$ b) понятна. Предположим, что $X^{*}$ сепарабельно. Докажем b) $\Rightarrow$ c). Заметим, что для банахова пространства $X$ следующие утверждения эквивалентны:

1) для $X$ имеется сепарабельная граница;

2) граница ext $B^{*}$ сепарабельна;

3) пространство $X^{*}$ сепарабельно.

Здесь импликации 3$) \Rightarrow 2$ ), 2) $\Rightarrow 1$ ) понятны (в линейном нормированном пространстве сепарабельность множества наследуется его произвольными подмножествами), а первое условие влечет последнее в силу известной теоремы Годфроя-Роде (см., например, [25, теорема 3.122]), согласно которой $B^{*}=\overline{\text { conv }}\|\cdot\| A$ (здесь $A$ - сепарабельная граница для $X$ ). Как следствие, шар $B^{*}$ сепарабелен, а значит, пространство $X^{*}$ также сепарабельно.

Далее, нам потребуется понятие (I)-генерирующего множества, введенного В. П. Фонфом и Й. Линденштрауссом. По определению множество $C \subset B^{*}$ (I)-генерирует шар $B^{*}$, если

$$
B^{*}=\overline{\operatorname{conv}}\left(\bigcup_{i} \overline{\operatorname{conv}} w^{*} C_{i}\right)
$$

при любом представлении $C=\bigcup C_{i}$ в виде объединения счетного семейства множеств $C_{i}$. В данном определении буква I происходит от латинского "intermedius" и объясняется тем, что

$$
B^{*}=\overline{\operatorname{conv}} C \Longrightarrow C(\mathrm{I}) \text {-генерирует } B^{*} \Longrightarrow B^{*}=\overline{\operatorname{conv}} w^{*} C .
$$

Положим $C_{i}:=\left\{f_{1}, \ldots, f_{i}\right\}, i \in I$ (где $F:=\left(f_{i}\right)_{i \in I}$ - набор функционалов из определения класса $\left.\left(\mathrm{Ex}-w^{*} \mathrm{~s}\right)\right)$. Ясно, что $F=\bigcup C_{i}$. Далее,

$$
B^{*}=\overline{\mathrm{conv}}\|\cdot\| \operatorname{ext} B^{*}
$$

по теореме Кадеца-Фонфа-Годфроя-Роде. Поскольку $X^{*}$ сепарабельно, то граница ext $B^{*}$ также сепарабельна. Далее, ext $S^{*} \subset \bar{F}^{w^{*}}$ по определению класса $\left(\mathrm{Ex}-w^{*} \mathrm{~s}\right)$, следовательно, ext $S^{*} \subset\left(\bigcup_{i} \overline{\operatorname{conv}} w^{*} C_{i}\right)$. Окончательно, $F$ (I)-генерирует шар $B^{*}$ в силу (3.5) и (3.4).

Теперь осталось воспользоваться одним результатом, полученным независимо О. Нигардом [23] и О.Ф.К. Календой [24], который утверждает, что если $C \subset B^{*}(\mathrm{I})$-генерирует шар $B^{*}$, то $C$ - множество Рейнуотера, т. е. множество со свойством: если ограниченная последовательность $\left(x_{n}\right) \subset X$ сходится поточечно на $C$, то $\left(x_{n}\right)$ сходится слабо. Предложение доказано. 


\section{§4. Основные результаты}

Основными результатами работы являются следующие.

ТеОрема 4.1. Пусть $X$ - банахово пространство из класса $(\mathrm{MeI}) \cap\left(\mathrm{Ex}-w^{*} \mathrm{~s}\right)$ (в частности, $X$ - сепарабельное банахово пространство), и пусть множество $M \subset X$ - замкнуто и т-связно. Предположим, что выполнено хотя бы одно из условий:

a) $M$ ограниченно компактно (в норме $\|\cdot\|$ пространства $X)$;

b) $M|\cdot|$-замкнуто $и \mathrm{~m}(x, y)|\cdot|$-компактно для любых $x, y \in X$;

c) $\mathrm{m}(x, y)\|\cdot\|$-компактно для любых $x, y \in X$.

Тогда $M$ монотонно линейно связно. Если, кроме того, $M$ ограниченно компактно, то $M$ P- и B-клеточноподобно, $P$ - и B-ациклично (относительно любой непрерывной теории (ко)гомологий) и является солнцем. Если $X$ конечномерно, то $M$ вдобавок $P$ - и В-стягиваемо.

В [26] показано, что множество $M \subset X$ обладает непрерывной мультипликативной (аддитивной) $\varepsilon$-выборкой из метрической проекции для всех $\varepsilon>0$ тогда и только тогда, когда оно является $\stackrel{\circ}{B}$-стягиваемым ( $B$-стягиваемым в случае аппроксимативной компактности $M)$. С учетом этого из теоремы 4.1 вытекает, что метрическая проекция на т-связное (монотонно линейно связное) замкнутое подмножество конечномерного пространства обладает непрерывной мультипликативной (аддитивной) $\varepsilon$-выборкой для всех $\varepsilon>0$. По-видимому, можно надеяться, что аналогичный результат верен и в бесконечномерном случае.

Для пространств с линейной вкладываемостью шаров [8] (в частности, в пространствах $\ell^{1}(n), C(Q)$, где $Q$ - метрический компакт, и $\left.C_{0}(Q)\right)$ теорема 4.1 частично усиливает результаты М. В. Балашова и Г. Е. Иванова $[27$, теорема 2.9 , лемма 4.18] о линейной связности $R$-слабо выпуклых (по Виалю) множеств: в пространствах с линейной вкладываемостью шаров пересечение $R$-слабо выпуклого множества с замкнутым или открытым шаром m-связно $[8$, теоремa 4.1].

Из теоремы 4.1 вытекает

СЛЕДСТвИЕ 4.1. Пусть $X$ - банахово пространство из класса (MeI) $\cap$ (Ex- $\left.w^{*} \mathrm{~s}\right)$, и пусть множество $M \subset X$ замкнуто. Предположим, что для некоторой точки $x \notin$ мннжество ее ближайших точек $P_{M} x$ компактно и m-связно. Тогда $P_{M} x$ монотонно линейно связно, $P$ - и $B$-клеточноподобно, $P$ и В-ациклично и является солнцем.

Отметим, что если $M \subset X \in(\mathrm{MeI})$ m-связно, то $P_{M} x$ также m-связно.

Действительно, пусть $u, v \in P_{M} x$. Поскольку $M$ m-связно, найдется $z \in$ $\mathrm{m}(u, v) \cap M, z \neq u, v$. Предположим, что $z \notin S(x,\|x-u\|)$. Поскольку $\operatorname{ext} S^{*}$ является границей для $X$, любую точку вне замкнутого шара (в нашем случае $B(x,\|x-u\|))$ всегда можно отделить от него экстремальным функционалом. Соответственно, $z \notin \llbracket u, v \rrbracket$. Получаем противоречие, поскольку $z \in \mathrm{m}(u, v)$, а в $X \in(\mathrm{MeI})$ всегда выполнено $\mathrm{m}(\cdot, \cdot)=\llbracket \cdot, \cdot \rrbracket$.

Для слабо компактных множеств имеет место

Теорема 4.2. Пусть $X$ - сепарабельное банахово пространство, и пусть

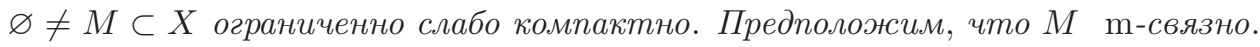
Тогда $M$ монотонно линейно связно. 
До сих пор остается открытым следующий вопрос. Хорошо известен следующий результат Власова [6]: в банаховом пространстве Р-ацикличное ограниченно компактное множество является солнцем ${ }^{2}$. Было бы интересно, особенно учитывая теорему 4.2, распространить теорему Власова на случай ограниченно слабо компактных множеств хотя бы в сепарабельном случае. Проблема здесь в следующем: несмотря на то, что слабо компактное подмножество $M$ сепарабельного банахова пространства метризуемо, а топология, порождаемая метрикой на $M$, совпадает со слабой топологией на $M$, теорема Эйленберга-Монтгомери не применима, поскольку метрическая проекция на $M$ полунепрерывна сверху лишь сильно-слабо (носитель один, а топологии на нем разные). Отметим, что в общем случае также не решена задача о солнечности слабо компактных чебышевских множеств.

\section{§5. Доказательства}

ДоказАТЕЛьСтво теоремы 4.1. Первое утверждение теоремы доказывается при использовании некоторых схем из работы [9]. Монотонная линейная связность установлена в [3], а оставшиеся утверждения теоремы следуют из леммы 5.2, которая будет доказана ниже. Конечномерный случай вытекает из п. а) и теоремы Брауна [28], согласно которой пересечение m-связного замкнутого подмножества конечномерного $X$ с замкнутым шаром $n$-связно для всех $n \in \mathbb{Z}_{+}$(т. е. каждое отображение во множество из $k$-сферы, $k \leqslant n$, непрерывно продолжается до отображения из $(k+1)$-мерного шара). Отсюда и из известной характеризации абсолютных ретрактов (см., например, [29, теорема 11.1]) вытекает, что $M$ стягиваемо и локально стягиваемо. Отметим [4], что условие b) теоремы 4.1 a fortiori выполнено в пространстве $X=c_{0}$.

Нам потребуется следующий вспомогательный результат.

Напомним, что множество $M$ называется метрически выпуклым или $d$-въnуклым (по Менгеру) [30] относительно метрики $d$, если для любых различных точек $x, y \in M$ множество $M \backslash\{x, y\}$ содержит точку $z$, находящуюся между $x$ и $y$ относительно метрики $d$, т. е. $d(x, y)=d(x, z)+d(z, y)$. Следующий результат принадлежит Менгеру [30] (см. также [31, с. 24]).

Лемма 5.А. Пусть $(Y, d)$ - выпуклое по Менгеру полное метрическое пространство. Тогда для любых $x, y \in Y$ существует изометрия $f:[0, d(x, y)] \rightarrow Y$ такая, что $f(0)=x$ и $f(d(x, y))=y$. Как следствие, $Y$ линейно связно.

ДокАЗАТЕЛЬСтво теоРемы 4.2. Достаточно рассмотреть ограниченное множество $M$. Хорошо известно (см., например, [25, предложение 3.107]), что слабо компактное подмножество банахова пространства с $w^{*}$-сепарабельным сопряженным метризуемо, причем топология, порождаемая метрикой на $M$, совпадает со слабой топологией на $M$. Также известно, что слабо компактное

\footnotetext{
2 Доказательство теоремы Власова опирается на классическую теорему ЭйленбергаМонтгомери о неподвижной точке (см., например, [13, следствие (32.12)]), при этом ацикличность понимается в смысле групп гомологий Александрова-Чеха с коэффициентами в поле (в работе Эйленберга-Монтгомери ацикличность понимается в смысле циклов Вьеториса и групп гомологий над полем коэффициентов). Однако гомологии Чеха изоморфны гомологиям Вьеториса на категории компактных метризуемых пространств, а понятия гомологической и когомологической ацикличности совпадают, если коэффициенты группы гомологий (когомологий) лежат в поле и носитель компактен.
} 
подмножество банахова пространства слабо полно (секвенциально слабо полно). В силу предложения 3.1 в пространствах класса (Ex-w*s), а следовательно, и в любых сепарабельных пространствах [5] $w$-полнота влечет $|\cdot|$-полноту относительно ассоциированной по Брауну нормы $|\cdot|$. Теперь для применения леммы 5.А осталось заметить, что в сепарабельном пространстве m-связность множества эквивалентна его $|\cdot|$-выпуклости (см. лемму 3.1).

Итак, по лемме 5.А любые две точки из $M$ соединяются дугой $k(\cdot)$, осуществляющей изометрию. Применяя лемму 3.1 , получаем, что $f_{i}(k(t))$ является монотонной функцией по $t$ для каждого $i \in I$. Пусть теперь $f \in \operatorname{ext} S^{*}$. Поскольку пространство $X$ сепарабельно, существует подпоследовательность $\left(f_{i}\right)$ из $F, w^{*}$-сходящаяся к $f$. Поэтому функция $f(k(t))$ также является монотонной. Теорема доказана.

Пусть пространство $X$ лежит в классе $(\mathrm{MeI}) \cap\left(\mathrm{Ex}-w^{*} \mathrm{~s}\right)$, и пусть $F=\left(f_{i}\right) \subset$ $\operatorname{ext} S^{*}-w^{*}$-плотное в ext $S^{*}$ семейство из определения класса (Ex- $\left.w^{*} \mathrm{~s}\right)$. Для каждого $n \in \mathbb{N}$ рассмотрим ограниченный линейный оператор $s_{n}: X \rightarrow \ell^{\infty}(n)$, определенный следующим образом:

$$
s_{n}(x)=\left(f_{1}(x), \ldots, f_{n}(x)\right) .
$$

Отметим, что всегда $\left\|s_{n}(x)\right\| \leqslant\|x\|$ и $\left\|s_{n}(x)\right\| \rightarrow\|x\|$ при $n \rightarrow \infty$. Последнее неравенство следует из того, что $\exp S^{*}$ является границей Джеймса для пространства $X$, т. е. $\|x\|=\max \left\{f(x) \mid f \in \operatorname{ext} S^{*}\right\}$.

Первое утверждение в следующей лемме доказывается небольшим видоизменением рассуждений из работы [9].

Лемма 5.1. Пусть $X$ - банахово пространство из класса $(\mathrm{MeI}) \cap\left(\mathrm{Ex}-w^{*} \mathrm{~s}\right)$ (в частности, $X$ - сепарабельное банахово пространство), и пусть $\varnothing \neq M \subset X$ ограниченно компактно. Тогда:

1) если $M \mathrm{~m}$-связно в $X$, mо $s_{n}(M)$ монотонно линейно связно в $\ell^{\infty}(n)$ для всех $n \in \mathbb{N}$;

2) если $s_{n}(M)$ m-связно в $\ell^{\infty}(n)$ для всех $n \in \mathbb{N}$, то $M$ монотонно линейно связно в $X$.

ДоказАтельство. 1) Достаточно рассмотреть случай, когда $M$ компактно. Требуемое утверждение для $s_{n}(M)$ следует из леммы 3.1 с учетом того, что m-связное подмножество конечномерного пространства монотонно линейно связно.

2) Предположим теперь, что $s_{n}(M)$ m-связно в $\ell^{\infty}(n)$ для всех $n$. Пусть $x, y \in M, x \neq y$. Отсюда следует, что существует $\nu>0$ такое, что если $n \geqslant \nu$, то $\left\|s_{n}(x)-s_{n}(y)\right\| \geqslant \varepsilon>0$. Поскольку $s_{n}(M)$ m-связно, выберем такую точку $z_{n} \in M$, что $s_{n}\left(z_{n}\right) \in \mathrm{m}\left(s_{n}(x), s_{n}(y)\right)$ и

$$
\left\|s_{n}\left(z_{n}\right)-s_{n}(x)\right\|=\left\|s_{n}\left(z_{n}\right)-s_{n}(y)\right\|=\frac{1}{2}\left\|s_{n}(x)-s_{n}(y)\right\| \geqslant \frac{\varepsilon}{2}>0 .
$$

Последнее равенство следует из непрерывности в силу леммы 5.А, примененной к замкнутому m-связному множеству $s_{n}(M)$. При $n \rightarrow \infty$ в силу компактности $M$ имеем, при необходимости переходя к подпоследовательностям, что $z_{n} \rightarrow z \in M$. Ясно, что $\left\|s_{n}\left(z_{n}-x\right)\right\| \rightarrow\|z-x\|$ и $\left\|s_{n}\left(z_{n}-y\right)\right\| \rightarrow\|z-y\|$. Отсюда $z \neq x, z \neq y$. Остается показать, что $z \in \mathrm{m}(x, y)$. При фиксированном $n \in \mathbb{N}$ из 
леммы 3.1 вытекает, что

$$
\min \left[f_{i}(x), f_{i}(y)\right] \leqslant f_{i}\left(z_{n}\right) \leqslant \max \left[f_{i}(x), f_{i}(y)\right], \quad i=1, \ldots, n,
$$

поскольку $s_{n}\left(z_{n}\right) \in \mathrm{m}\left(s_{n}(x), s_{n}(y)\right)$. При $n \rightarrow \infty$ получаем

$$
\min \left[f_{i}(x), f_{i}(y)\right] \leqslant f_{i}(z) \leqslant \max \left[f_{i}(x), f_{i}(y)\right], \quad i \in I,
$$

откуда $z \in \mathrm{m}(x, y)$ по лемме 3.1. Теперь монотонная линейная связность m-связного множества $M$ обеспечивается теоремой 4.1 .

Отметим, что п. 2) леммы 5.1 не используется при доказательстве теоремы 4.1 и следующей леммы.

Лемма 5.2. Пусть $X \in(\mathrm{MeI}) \cap\left(\mathrm{Ex}-w^{*} \mathrm{~s}\right)$ - банахово пространство (в частности, $X$ сепарабельно или $\left.X=\ell^{\infty}\right)$, и пусть множество $\varnothing \neq M \subset X$ ограниченно компактно и т-связно. Тогда $M P$ - и B-клеточноподобно, из множеств

$$
P_{M} x, \quad M \cap B(x, r), \quad x \in X, \quad r>0,
$$

клеточноподобно. В частности, множество $M P$ - и B-ациклично (относительно любой непрерывной теории (ко)гомологий).

Прежде чем переходить к доказательству леммы 5.2, напомним, что обратной системой топологических пространств (см., например, [32, с. 56]) называется семейство $\mathcal{S}=\left\{X_{\alpha}, \pi_{\alpha}^{\beta}, \Sigma\right\}$, где множество $\Sigma$ частично упорядочено отношением $\prec, X_{\alpha}$ - топологическое хаусдорфово пространство и $\pi_{\alpha}^{\beta}: X_{\beta} \rightarrow X_{\alpha}-$ непрерывное отображение для любых $\alpha \prec \beta$, при этом $\pi_{\alpha}^{\alpha}=\mathrm{id}_{X_{\alpha}}$ и $\pi_{\alpha}^{\beta} \pi_{\beta}^{\gamma}=\pi_{\alpha}^{\gamma}$ для всех $\alpha \prec \beta \prec \gamma$. Следующее подпространство произведения $\prod_{\alpha \in \Sigma} X_{\alpha}$ называется обратным пределом системы $\mathcal{S}$ и обозначается

$$
\varliminf_{\lim } \mathcal{S}=\left\{\left(x_{\alpha}\right) \in \prod_{\alpha \in \Sigma} X_{\alpha} \mid \pi_{\alpha}^{\beta}\left(x_{\beta}\right)=x_{\alpha} \quad \forall \alpha \prec \beta\right\} .
$$

Если $\pi_{\alpha}: \lim \mathcal{S} \rightarrow X_{\alpha}$ - ограничение проекции $p_{\alpha}: \prod_{\alpha \in \Sigma} X_{\alpha} \rightarrow X_{\alpha}$ на ось $\alpha$ (каноническая проекция), то $\pi_{\alpha}=\pi_{\alpha}^{\beta} \pi_{\beta}$ для всех $\alpha \prec \beta$.

Нам потребуется следующий известный результат, в котором утверждение 1) содержится, например, в [15], а утверждение 2) получено в [33] (см. также [16]). При этом утверждение а) является следствием классической теоремы А.Н. Тихонова о компактности; утверждение b) хорошо известно и принадлежит А.Г. Курошу и Н.Е. Стинроду.

Лемма 5.В. 1) Пусть $\mathcal{S}=\left\{X_{\alpha}, \pi_{\alpha}^{\beta}, \Sigma\right\}$ - обратная система. Тогда предел $\lim _{\longleftarrow} \mathcal{S}$ является замкнутым подмножеством произведения $\prod_{\alpha \in \Sigma} X_{\alpha}$. Кроме того, выполнены следующие утверждения.

а) Если $X_{\alpha}$ компактно для всякого $\alpha \in \Sigma$, то предел $\lim _{\mathcal{S}}$ компактен;

b) Если $X_{\alpha}$ компактно и непусто для всякого $\alpha \in \Sigma \overleftarrow{, ~ т о ~} \lim _{\longleftarrow} \mathcal{S}$ компактно u непусто;

с) Если $X_{\alpha}$ является континуумом для всякого $\alpha \in \Sigma$, mо $\underset{\lim }{\longleftarrow}$ является континуумом;

d) Если $X_{\alpha}$ компактно и ациклично ${ }^{3}$ для всякого $\alpha \in \Sigma$, то предел $\lim \mathcal{S}$ компактен и ацикличен;

\footnotetext{
${ }^{3}$ Относительно любой непрерывной теории когомологий.
} 
е) Если $X_{\alpha}$ метризуемо для всякого $\alpha \in \Sigma$ и $\Sigma$ счетно, то предел $\lim _{\longleftarrow} \mathcal{S}$ метризуем.

2) Если $\mathcal{S}=\left\{X_{n}, \pi_{n}^{p}, \Sigma\right\}$ - счетная обратная система и каждое $X_{n}$ компактно и клеточноподобно, то обратный предел $\lim _{\mathcal{S}}$ также компактен и является клеточноподобным множеством.

ДокАЗАтельство ЛЕммы 5.2. Достаточно рассмотреть случай, когда $M$ компактно. Пусть $\Omega$ - семейство всех конечных подмножеств из $F \subset \operatorname{ext} S^{*}$. Поскольку множество конечных последовательностей натуральных чисел счетно, то $\Omega$ счетно. Множество $\Omega$ направлено по включению, т. е. направлено отношением $\prec$, определенным следующим образом: $A \prec B$ в том и только в том случае, когда $A \subset B$. Для $A \in \Omega$ определим отображение $s_{A}: X \rightarrow \ell^{\infty}(A)$,

$$
s_{A}(x)=\{f(x) \mid f \in A\} .
$$

Тогда $s_{A}$ - линейный ограниченный оператор. Если $A, B \in \Omega, A \subset B$, то имеется естественный оператор сужения $\ell^{\infty}(B) \rightarrow \ell^{\infty}(A)$ и, следовательно, имеется естественное отображение сужения $s_{B A}$. Хорошо известно, что семейство $\left\{s_{A} \mid A \in \Omega\right\}$ образует обратную систему по отношению к отображениям сужения. Согласно утверждению 1) леммы 5.1 множества $s_{A}(M)$ являются компактными т-связными подмножествами пространства $\ell^{\infty}(A)$ и, следовательно, по теореме Брауна [19, теорема 1] бесконечно связными (а значит, клеточноподобными и ацикличными множествами).

В теории обратных систем хорошо известна следующая конструкция. Пусть $(X, \pi)=\left(X_{\alpha}, \pi_{\alpha}^{\beta}, \Sigma\right)$ - обратная система множеств, а $Z$ - произвольное фиксированное множество. Пусть, далее, для каждого $\alpha \in \Sigma$ задано отображение $g_{\alpha}: Z \rightarrow X_{\alpha}$, причем эти отображения таковы, что $\pi_{\alpha}^{\beta} g_{\beta}=g_{\alpha}$ всякий раз, когда $\alpha \leqslant \beta$. Тогда оказывается, что существует (и притом единственное) отображение $g: Z \rightarrow X_{\infty}:=\lim (X, \pi)$, обладающее свойством $\pi_{\alpha} \circ g=g_{\alpha}$ при всех $\alpha$. При этом если $Z$ - топологическое пространство и все $g_{\alpha}-$ непрерывные отображения, то $g: Z \rightarrow X_{\infty}$ непрерывно.

Поскольку $\left\{s_{A} \mid A \in \Omega\right\}$ - обратная система, имеет место суперпозиция отображений $s_{A}=s_{B A} \circ s_{B}$ для $A, B \in \Omega, A \subset B$, где $s_{B A}$ - отображение сужения (см. [34, с. 428]). Отсюда отображение $g: M \rightarrow M_{\infty}:=\lim s_{A}(M)$ непрерывно (по изложенному выше) и инъективно (см. $[34,(6.9)])$. Докажем его сюръективность.

Предположим, что

$$
\left(x_{A}\right)_{A \in \Omega} \in \lim _{\longleftarrow} s_{A}(M) \subset \prod_{A \in \Omega} s_{A}(M) .
$$

Для каждого $A \in \Omega$ выберем $y_{A} \in M$ таким образом, что $s_{A}\left(y_{A}\right)=x_{A}$. Тогда $\left(y_{A}\right)_{A \in \Omega}$ является сетью (направленностью) в $M$ и вследствие компактности $M$ имеет предельную точку $y \in M$. Остается доказать, что $s_{A}(y)=x_{A}$ для каждого $A \in \Omega$. Рассмотрим $A, B \in \Omega, B \supset A$. Тогда $s_{A}\left(y_{B}\right)=\left.s_{B}\left(y_{B}\right)\right|_{A}=x_{A}$. Переходя к пределу по подсети, получаем, что $s_{A}(y)=x_{A}$.

Итак, отображение $g: M \rightarrow M_{\infty}$ непрерывно и по доказанному выше является биекцией. Хорошо известно [32, с. 169], что любое отображение компактного пространства в хаусдорфово замкнуто. Как следствие, непрерывное биективное отображение компактного пространства на хаусдорфово является гомеоморфизмом. Поскольку обратный предел $M_{\infty}$ хаусдорфов [32, с. 171], предыдущее утверждение применимо в нашей ситуации. Итак, $M$ гомеоморфно $M_{\infty}$. 
Далее, хорошо известно, что клеточноподобность (равно как ацикличность) является топологическим свойством [13, с. 439]. Поскольку $M \simeq M_{\infty}$, из леммы 5.В следует, что $M$ клеточноподобно (и, следовательно, ациклично), так как по упомянутой теореме Брауна каждое из $s_{A}(M)$ обладает таким свойством. При этом утверждение 2) леммы 5.В применимо в силу известного результата, состоящего в том, что множество конечных последовательностей натуральных чисел счетно. Лемма доказана.

Ответ на обратный вопрос о монотонной линейной связности $B$-ацикличных (P-ацикличных) множеств в общем случае неизвестен. Здесь стоит отметить, что для любого $n \geqslant 3$ можно построить пример конечномерного пространства $X_{n}$, в котором существует не монотонно линейно связное чебышевское множество (являющееся заведомо солнцем, а также $P$ - и $B$-ацикличным множеством). Действительно, рассмотрим пространства $X_{n}$ со свойством $\overline{\operatorname{ext}} S^{*}=S^{*}$. P.Р. Фелпс [35] показал, что свойство $\overline{\operatorname{ext}} S^{*}=S^{*}$ выполнено для пространства $X_{n}$, если и только если каждое выпуклое ограниченное замкнутое подмножество в $X_{n}$ представимо как пересечение замкнутых шаров (иными словами, $X_{n} \in(\mathrm{MIP})$, т. е. удовлетворяет свойству пересечения Мазура); как следствие, в таком пространстве монотонная линейная связность замкнутого множества равносильна его выпуклости. Далее, для любого $n \geqslant 3$ в [36] построен пример пространства $X_{n}^{\prime}$ со свойством $\overline{\operatorname{ext}} S^{*}=S^{*}$, содержащего неограниченное невыпуклое чебышевское множество $M^{\prime}$ (при этом любое ограниченное чебышевское множество в таком $X_{n}^{\prime}$ выпукло). Таким образом, $M^{\prime}$ служит примером не монотонно линейно связного В-ацикличного (Р-ацикличного) множества (чебышевского солнца).

В связи с этим же вопросом отметим, что Браун [1] ввел важный класс линейных нормированных пространств, так называемых (ВМ)-пространств (см. также [28], [37], [9]), которые оказались очень естественными в задаче об т-связности солнц. Пространства класса $(\mathrm{BM})$ (в частности, $\ell^{\infty}(n)$ и $\left.c_{0}\right)$ являются "хорошими", поскольку в них всякое ограниченно компактное солнце m-связно (см. [1], [4]) и, следовательно, монотонно линейно связно. Поскольку по теореме Власова ограниченно компактное $P$-ацикличное множество является солнцем, в таких пространствах $P$-ацикличность влечет монотонную линейную связность. Также отметим [38], что в конечномерном полиэдральном пространстве $X_{n}$ каждое солнце монотонно линейно связно, если и только если $X_{n} \in(\mathrm{BM})$. Соответственно, $\ell^{1}(n) \notin(\mathrm{BM}), n \geqslant 3$, и поэтому в $\ell^{1}(n)$ существует не монотонно линейно связное солнце. Однако не известно, будет ли такое солнце $P$-ацикличным (или даже хотя бы $P$-связным).

Автор благодарит И. Г. Царькова, А. А. Васильеву, Я.-Д. Хардтке, С. А. Богатого и У.Х. Каримова за обсуждения, а также рецензента за внимательное прочтение рукописи и полезные замечания.

\section{Список литературы}

1. A. L. Brown, "Suns in normed linear spaces which are finite dimensional", Math. Ann., 279:1 (1987), 87-101.

2. B. Brosowski, F. Deutsch, J. Lambert, P. D. Morris, "Chebyshev sets which are not suns", Math. Ann., 212:2 (1974), 89-101. 
3. А.Р. Алимов, "Монотонная линейная связность чебышевских множеств в пространстве $C(Q)$ ", Матем. сб., 197:9 (2006), 3-18; англ. пер.: A. R. Alimov, "Monotone path-connectedness of Chebyshev sets in the space $C(Q)$ ", Sb. Math., 197:9 (2006), 1259-1272.

4. А. Р. Алимов, "Связность солнц в пространстве $c_{0}$ ", Изв. РАН. Сер. матем., 69:4 (2005), 3-18; англ. пер.: A. R. Alimov, "Connectedness of suns in the space $c_{0}$ ", Izv. Math., 69:4 (2005), 651-666.

5. А.Р. Алимов, “Локальная солнечность солнц в линейных нормированных пространствах", Фундамент. и прикл. матем., 17:7 (2012), 3-14; англ. пер.: A. R. Alimov, "Local solarity of suns in normed linear spaces", J. Math. Sci., 197:4 (2014), 447-454.

6. Л. П. Власов, "Аппроксимативные свойства множеств в линейных нормированных пространствах", УМH, 28:6(174) (1973), 3-66; англ. пер.: L. P. Vlasov, "Approximative properties of sets in normed linear spaces", Russian Math. Surveys, 28:6 (1973), 1-66.

7. А.Р. Алимов, "Монотонно линейно связное чебышевское множество является солнцем", Матем. заметки, 91:2 (2012), 305-307; англ. пер.: A. R. Alimov, "A monotone path connected Chebyshev set is a sun", Math. Notes, 91:2 (2012), 290-292.

8. A. R. Alimov, "Monotone path-connectedness of $R$-weakly convex sets in spaces with linear ball embedding", Eurasian Math. J., 3:2 (2012), 21-30.

9. C. Franchetti, S. Roversi, Suns, $M$-connected sets and P-acyclic sets in Banach spaces, Preprint № 50139, Inst. di Mat. Appl. "G. Sansone", 1988.

10. С. А. Мелихов, "Стинродовские гомотопии", УМH, 64:3(387) (2009), 73-166; англ. пер.: S. A. Melikhov, "Steenrod homotopy", Russian Math. Surveys, 64:3 (2009), 469-551.

11. W.S. Massey, Homology and cohomology theory, Monogr. Textbooks Pure Appl. Math., 46, Marcel Dekker, Inc., New York-Basel, 1978, xiv+412 pp.

12. K. Eda, U.H. Karimov, D. Repovš, "On (co)homology locally connected spaces", Topology Appl., 120:3 (2002), 397-401.

13. L. Górniewicz, Topological fixed point theory of multivalued mappings, 2nd ed., Topol. Fixed Point Theory Appl., 4, Springer, Dordrecht, 2006, xiv+539 pp.

14. L. Górniewicz, "Topological structure of solution sets: current results", Arch. Math. (Brno), 36 (2000), 343-382.

15. R. Dragoni, J.W. Macki, P. Nistri, P. Zecca, Solution sets of differential equations in abstract spaces, Pitman Res. Notes Math. Ser., 342, Harlow, Longman, 1996, xii+100 pp.

16. J. Andres, G. Gabor, L. Górniewicz, "Acyclicity of solution sets to functional inclusions", Nonlinear Anal., 49:5 (2002), 671-688.

17. W. Kryszewski, "On the existence of equilibria and fixed points of maps under constraints", Handbook of topological fixed point theory, Springer, Dordrecht, 2005, 783-866.

18. Shouchuan Hu, N.S. Papageorgiou, Handbook of multivalued analysis, v. II: Applications, Math. Appl., 500, Kluwer Academic Publishers, Dordrecht, 2000, xii+926 pp.

19. A. L. Brown, "Chebyshev sets and the shapes of convex bodies", Methods of functional analysis in approximation theory (Bombay, 1985), Internat. Schriftenreihe Numer. Math., 76, Birkhäuser, Basel, 1986, 97-121.

20. B. Brosowski, F. Deutsch, "On some geometric properties of suns", J. Approximation Theory, 10:3 (1974), 245-267.

21. J.R. Giles, "The Mazur intersection problem", J. Convex Anal., 13:3-4 (2006), 739-750. 
22. E. N. Dancer, B. Sims, "Weak star separability", Bull. Austral. Math. Soc., 20:2 (1979), 253-257.

23. O. Nygaard, "A remark on Rainwater's theorem", Ann. Math. Inform., 32 (2005), $125-127$.

24. O.F.K. Kalenda, "( $I)$-envelopes of unit balls and James' characterization of reflexivity", Studia Math., 182:1 (2007), 29-40.

25. M. Fabian, P. Habala, P. Hájek, V. Montesinos, V. Zizler, Banach space theory. The basis for linear and nonlinear analysis, CMS Books Math./Ouvrages Math. SMC, Springer, New York, 2011, xiv+820 pp.

26. И.Г. Царьков, "Геометрическая теория приближений в работах Н.В. Ефимова и С. Б. Стечкина", Соврем. Пробл. Матем. Механ., 4:2 (2011), 57-78.

27. М. В. Балашов, Г.Е. Иванов, "Слабо выпуклые и проксимально гладкие множества в банаховых пространствах", Изв. РАН. Сер. матем., 73:3 (2009), 23-66; англ. пер.: M. V. Balashov, G. E. Ivanov, "Weakly convex and proximally smooth sets in Banach spaces", Izv. Math., 73:3 (2009), 455-499.

28. A. L. Brown, "On the connectedness properties of suns in finite-dimensional spaces", Workshop/Miniconference on functional analysis and optimization (Canberra, 1988), Proc. Centre Math. Anal. Austral. Nat. Univ., 20, Austral. Nat. Univ., Canberra, 1988, 1-15.

29. Sze-Tsen Hu, Theory of retracts, Wayne State Univ. Press, Detroit, 1965, 234 pp.

30. K. Menger, "Untersuchungen über allgemeine Metrik", Math. Ann., 100:1 (1928), $75-163$.

31. K. Goebel, W. A. Kirk, Topics in metric fixed point theory, Cambridge Stud. Adv. Math., 28, Cambridge Univ. Press, Cambridge, 1990, viii+244 pp.

32. Encyclopedia of general topology, eds. K. P. Hart, J. Nagata, J. E. Vaughan, Elsevier, Amsterdam, 2004, x+526 pp.

33. G. Gabor, "On the acyclicity of fixed point sets of multivalued maps", Topol. Methods Nonlinear Anal., 14:2 (1999), 327-343.

34. J. Dugundji, Topology, Allyn and Bacon, Inc., Boston, MA, 1966.

35. R. R. Phelps, "A representation theorem for bounded convex sets", Proc. Amer. Math. Soc., 11:6 (1960), 976-983.

36. И.Г. Царьков, "Ограниченные чебышевские множества в конечномерных банаховых пространствах", Матем. заметки, 36:1 (1984), 73-87; англ. пер.: I. G. Tsar'kov, "Bounded Chebyshev sets in finite-dimensional Banach spaces", Math. Notes, 36:1 (1984), 530-537.

37. A. L. Brown, "On the problem of characterising suns in finite dimensional spaces", Proceedings of the Fourth international conference on functional analysis and approximation theory, v. I (Potenza, 2000), Rend. Circ. Mat. Palermo (2) Suppl., 68, part I, 2002, 315-328.

38. A.L. Brown, "Suns in polyhedral spaces", Seminar of mathematical analysis (Malaga/Seville, 2002/2003), Colecc. Abierta, 64, Univ. Sevilla Secr. Publ., Seville, 2003, 139-146.

АлЕКСеЙ РОстислАвович АлИмов

Поступило в редакцию

(Alexey R. Alimov)

15.05 .2013

Московский государственный университет

18.10 .2013

им. М. В. Ломоносова,

механико-математический факультет

E-mail: alexey.alimov@gmail.com 